\title{
A constitutive model for the compressive response of metallic closed-cell foams including micro-inertia effects
}

\author{
Romain Barthélémy $^{1,2, a}$, Nicolas Jacques ${ }^{2}$, François Vermeersch ${ }^{1}$, and Steven Kerampran ${ }^{2}$ \\ ${ }^{1}$ MBDA France, 1 avenue Réumur, 92350 Le Plessis-Robinson, France \\ 2 ENSTA Bretagne, LBMS, 2 rue François Verny, 29800 Brest, France
}

\begin{abstract}
Metallic foams have known a keen interest in the last decades. Their ability to undergo very large deformations while transmitting low stress levels make them capable of performing functions of protective layers against intense loadings and of energy absorbers, for instance. The behaviour of metal foams varies considerably between quasi-static and dynamic regimes. Those differences can be linked to the strain-rate sensitivity of the skeleton material and to micro-inertial effects (induced by the crushing of the foam cells).

In the present work, a micromechanical model has been developed to take into account micro-inertia effects on the macroscopic behaviour of closed-cell foams under dynamic loading conditions. The proposed modelling is based on the dynamic homogenisation procedure introduced by Molinari and Mercier (J. Mech. Phys. Solids 49 (2001) 1497-1516). Within this framework, the macrostress is the sum of two terms. The first one is a static stress, that can be described with any existing model of metal foam. The second contribution is a dynamic stress related to micro-inertia effects. Considering an initially spherical shell as a Representative Volume Element (RVE) of the foam material, a closed-form expression of the dynamic stress was obtained. The proposed modelling was applied to shock propagation in aluminium foams (it should however be noted that the present theory is not restricted to uniaxial deformation but can be applied to arbitrary loadings). From experimental data of the literature, it is observed that incorporating micro-inertia effects allows one to achieve a better description of the foam shock response. This indicates that micro-inertia may have a significant influence on the dynamic behaviour of metallic foams.
\end{abstract}

\section{Introduction}

Among recent materials, cellular ones have known large developments in the last decades. They are found at natural state (wood, cancellous bones, sponges, cork...) or manmade (bread, foams...). Cellular solids are often classified according to their relative density (the ratio of the density of the foam sample to the one of its constitutive material). Cellular materials with high relative density are called "porous media" while "foam" corresponds to materials with a relative density lower than 0.3 [1]. The latter is the focus of this study.

Foam skeletons can be made of different materials such as polymer, ceramic, glass or metal and present two types of structure (open-cell or closed-cell), see Fig. 1.

Metallic foams have many attractive features which make them reliable for applications such as vibration suppression, heat transfer, electromagnetic shielding or blast protection [2]. The last application, which is of our concern, involves high strain-rate deformation.

The quasi-static behaviour of metal foams is well-known and documented [3-9]. Some studies deal with the high strain-rate response of cellular materials [10-12]. A common conclusion of these studies is that metal foams exhibit some strain-rate sensitivity. One can also find, in literature, studies regarding the origins of this phenomenon

${ }^{a}$ Corresponding author: romain. barthelemy@mbdasystems.com
[13-15]. Zhao \& al. [16] give four elements susceptible to explain the dependency of metallic foams to strain-rates:

- the rise in pressure of the internal fluid (particularly for closed-cell foams);

- the strain-rate sensitivity of the constitutive material of the skeleton;

- the shock-enhancement mechanism [14];

- micro-inertia effects appearing when foam cells are rapidly crushed.

The influence of the entrapped gas is negligible in the case of metal foam (because of the high strength of the skeleton material). Material strain-rate sensitivity (viscoplasticity) and the shock enhancement mechanism can be easily accounted for by numerical simulations using standard explicit codes. This is not the same for micro-inertia effects. Indeed, the role of this mechanism is not fully understood and, to our knowledge, constitutive models accounting for microscale inertia have been developed only for open-cell foams [17].

The aim of the present work is to investigate the influence of micro-inertia on the shock response of closed-cell metal foams. For this purpose, a new model based on micromechanics has been developed. It will be shown that taking micro-inertia effects into account when modelling aluminium foams leads to a better agreement with published experimental data.

This is an Open Access article distributed under the terms of the Creative Commons Attribution License 4.0, which permits unrestricted use, distribution, and reproduction in any medium, provided the original work is properly cited. 

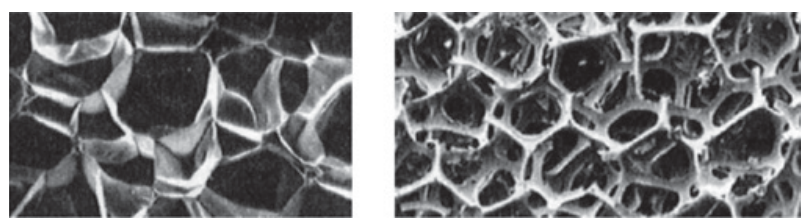

Figure 1. Microstructures of a closed-cell (on the left) and an open-cell (on the right) metallic foam [1].

\section{Modelling of metal foam behaviour under uniaxial compression}

\subsection{Micro-inertia effects}

To account for micro-inertia effects, an original model is proposed. This model has been developed using a micromechanical analysis and the dynamic homogenization procedure introduced by Molinari and Mercier [18] (see also Sartori \& al. [19]). The aim of this procedure is to link microscopic quantities (written in lower case letters hereafter) to macroscopic ones (written in capital letters) through averaging over a Representative Volume Element (RVE). In the present case, the RVE is chosen to be an initially spherical shell.

Within the framework proposed by Molinari and Mercier [18], the macroscopic (nominal) stress tensor $\mathrm{P}$ is defined as:

$$
\underline{\underline{P}}=<\underline{\underline{p}}>_{0}+<\rho_{0} \underline{\ddot{u}} \otimes \underline{x_{0}}>_{0},
$$

where $\rho_{0}$ denotes the initial density of the foam skeleton material, $x_{0}$ is the initial position of a material point in the RVE and $\underline{\ddot{u}}$ its acceleration. $<.>_{0}$ stands for the averaging operator:

$$
<X>_{0}=\frac{1}{\Omega_{0}} \int_{\Omega_{0}} X \cdot d \Omega_{0} .
$$

As discussed in [19], Eq. (1) is not appropriate to obtain a closed-form expression of the macrostress as the microscopic acceleration and stress field are in general unknown. A more convenient way to characterize the macroscopic response of the material is to use the virtual work principle, combined with an approximate trial velocity field. Indeed, the macroscopic stress (as defined by Eq. (1)) is conjugated with the macroscopic velocity gradient $\underline{F}$ through the virtual work principle:

$$
\delta \underline{\underline{F}}^{T}: \underline{\underline{P}}=<\delta \underline{\underline{f}}^{T}: \underline{\underline{p}}>_{0}+<\rho_{0} \delta \underline{u} . \underline{\ddot{u}}>_{0} .
$$

From this relation, the macrostress can be written as the sum of two components, a static stress $P^{\text {sta }}$ related to the constitutive response of the skeleton $\overline{\overline{\text { material }}}$ and a dynamic (or inertial) stress $P^{d y n}$ induced by the local acceleration of the material within the RVE:

$$
\underline{\underline{P}}=\underline{\underline{P^{s t a}}}+\underline{\underline{P^{d y n}}} \text {. }
$$

We consider as a Representative Volume Element of the foam material an initially spherical shell of radius Rc and the following acceleration and virtual displacement fields are considered:

$$
\underline{\ddot{u}}=\underline{\underline{F}} \cdot \underline{x_{0}} \quad \text { and } \quad \delta \underline{u}=\delta \underline{\underline{F}} \cdot \underline{x_{0}} .
$$

Finally, the following expression for the dynamic stress is obtained:

$$
\underline{\underline{P^{d y n}}}=\frac{\overline{\rho_{0}}}{3} \cdot R_{c}^{2} \cdot \underline{\underline{F}}^{T},
$$

where $\overline{\rho_{0}}$ is the initial average mass density of the foam material. We observe that the dynamic stress also depends on the initial radius $R_{c}$ of the foam cells. This quantity can be identified from micrographic observations of foam microstructure. More details about the derivation of the micro-inertia model will be given in a future paper.

Because the present paper is focused on the propagation of planar shocks, we consider in the following a uniaxial deformation state of the form:

$$
\underline{\underline{F}}=\left[\begin{array}{lll}
F & & \\
& 1 & \\
& & 1
\end{array}\right] \text {. }
$$

In this case, the component of the (nominal or Cauchy) stress in the shock propagation direction is given by:

$$
\Sigma^{d y n}=P^{d y n}=\frac{\overline{\rho_{0}}}{3} \cdot R_{c}^{2} \cdot \ddot{F} .
$$

\subsection{Static response (uniaxial deformation)}

In the present study, we do not intend to develop a micromechanical model to describe the quasi-static stress. Indeed, the static behaviour of foam materials can be easily characterized using standard experimental techniques. For this reason, it is believed that it is better to employ a phenomenological constitutive model.

The quasi-static compressive behaviour of metal foams generally exhibits three phases: an elastic one observed in the early deformation, a "plateau" regime in which the stress is almost constant and a densification (or hardening) phase with a strong increase in stress. Several simplified models exist in the literature (see the Rigid Perfectly Plastic Locking model [20] and the Elastic Perfectly Plastic Hardening model [21] for example). In the present work, an elastic-plastic model with non-linear isotropic hardening has been used. This model is based on the following hypothesis:

- the logarithmic strain $\epsilon=\ln (F)$ is used;

- the strain is described as the sum of an elastic $\left(\epsilon_{e}\right)$ and a plastic $\left(\epsilon_{p}\right)$ part;

- the elastic strain is calculated by using the following linear relation $\epsilon_{e}=\Sigma / E^{*}$;

- the plastic part $\dot{\epsilon}_{p}$ evolves as $\dot{\epsilon}_{p}=\dot{p} \cdot \operatorname{sgn}(\Sigma)$;

- the effective plastic strain $\dot{p}$ is defined by the KuhnTucker complementary conditions [22]:

$$
\dot{p} \geq 0, \quad|\Sigma|-\Sigma_{y} \leq 0, \quad\left(|\Sigma|-\Sigma_{y}\right) \cdot \dot{p}=0 ;
$$

- the evolution of the yield stress $\Sigma_{y}$ of the foam as a function of the effective plastic strain $p$ is given by the following relationships:

$$
\begin{aligned}
\Sigma_{y}=\Sigma_{p l} & \text { if } p \leq \epsilon_{d}(10) \\
\Sigma_{y}=\Sigma_{p l}+K_{p} \cdot\left(p-\epsilon_{d}\right)^{n} \cdot \frac{\epsilon_{l}-\epsilon_{d}}{\epsilon_{l}-p} & \text { if } p>\epsilon_{d} .
\end{aligned}
$$


Table 1. Parameters of the elastic plastic model with non linear hardening corresponding to an Alporas ${ }^{\circledR}$ foam of initial relative density 0.11

\begin{tabular}{|c||c|c|}
\hline Parameter's name & Description & Value \\
\hline \hline$E^{*}$ & Elastic modulus & $3.120 \mathrm{GPa}$ \\
$\Sigma_{p l}$ & Initial yield stress & $3 \mathrm{MPa}$ \\
$\epsilon_{d}$ & Densification strain & 0.6328 \\
$K_{p}$ & Hardening modulus & $24.6 \mathrm{MPa}$ \\
$n$ & Hardening exponent & 2.11 \\
$\epsilon_{l}$ & Locking strain & 4.16 \\
\hline
\end{tabular}

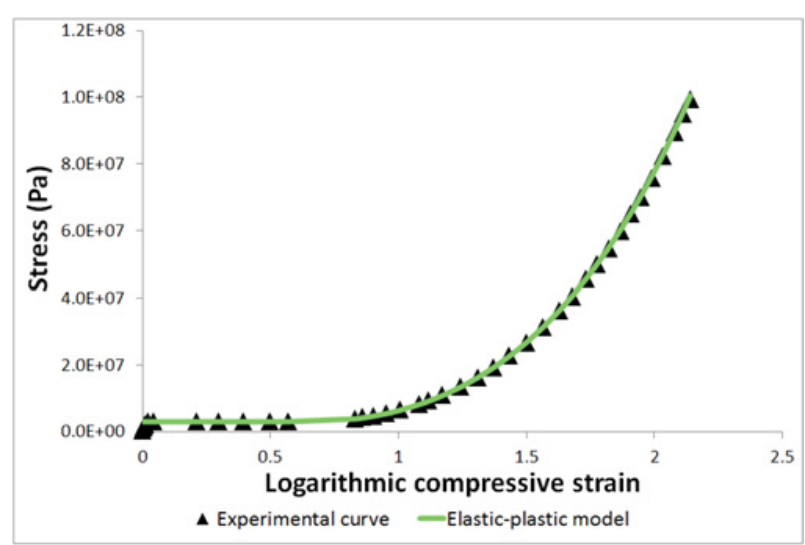

Figure 2. Experimental quasi-static compressive stress-strain curve of an Alporas ${ }^{\circledR}$ foam of initial relative density 0.11 [23] along with the prediction of the elastic-plastic model with nonlinear hardening.

The considered model involves six parameters which are the elastic modulus $E^{*}$, the initial yield stress $\Sigma_{p l}$, the densification strain $\epsilon_{d}$, the hardening modulus $K_{p}$, the hardening exponent $n$ and the locking strain $\epsilon_{l}$. We have identified these parameters for a closed-cell aluminium foam of initial density 0.11 (Alporas ${ }^{\circledR}$ foam produced by Shinko Wire Co. Ltd.) using the experimental data of Radford et al. [23].

These authors conducted uniaxial compressive tests on cylindrical foam samples at a nominal strain-rate of $10^{-3} \mathrm{~s}^{-1}$. The identification of the model parameters was carried out using a least-square fitting technique. The obtained parameters are presented in Table 1. Figure 2 shows a comparison between the experimental stress-strain curve and the prediction based on the elastic plastic model with non linear hardening.

\section{Results}

\subsection{Presentation of the considered configuration}

In order to assess the accuracy of the proposed model, we have used the results of the impact tests carried out by Radford et al. [23]. The experimental set-up is presented in Fig. 3: a cylindrical foam projectile is launched by a gas gun and impacts a steel bar initially at rest. The foam samples had a diameter of $28 \mathrm{~mm}$ and a length of 50 or $100 \mathrm{~mm}$. Impact velocities ranging from $50 \mathrm{~m} \mathrm{~s}^{-1}$ to $500 \mathrm{~m} \mathrm{~s}^{-1}$ were considered. At the impact against the bar, a shock wave is generated and propagates across the

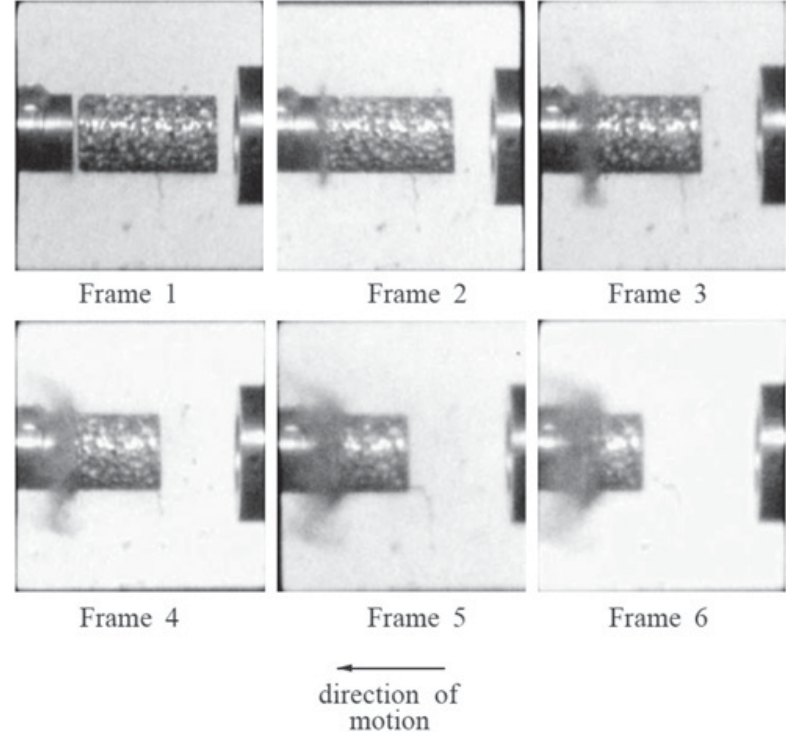

Figure 3. Photographic sequence of the impact of an Alporas ${ }^{\circledR}$ sample against a steel bar at about $380 \mathrm{~m} \mathrm{~s}^{-1}$. Inter-frame time: $20 \mu$ s. From [23].

foam sample. Strain gauges were mounted on the bar at about $285 \mathrm{~mm}$ of the impact surface. The authors deduced the time-evolution of the contact pressure from the strain gauge recordings.

From Fig. 3, no lateral expansion of the specimen is observed during the shock compaction of the sample. This behaviour can be related to the results presented by Deshpande and Fleck [24], who observed that the plastic Poisson ratio of low-density metal foams is close to zero.

\subsection{One-dimensional finite element simulations}

Standard finite element dynamic codes do not account for micro-inertia effects. For this reason, we have developed a one-dimensional finite element code in Fortran 90 to simulate the impact tests described in the previous section. Standard linear finite elements are used to approximate the displacement and velocity fields. The time integration is based on the central difference scheme.

It is worth emphasizing that the present simulations do not involve any adjustable parameters. Indeed, the parameters related to the static response of the foam material were identified from a single quasi-static compression curve (see Sect. 2.2). The dynamic stress (micro-inertia effects) depends on the radius $R_{c}$ of the cells of the foam microstructure (in the modelling, all cells are assumed to have the same size). From micrographic observations, Radford et al. [23] indicated that the average cell size of the Alporas ${ }^{\circledR}$ foam is about $4 \mathrm{~mm}$. Thus, we have taken $R_{c}=2 \mathrm{~mm}$.

In the computations, the output bar is not meshed, but is modelled using an impedance boundary condition (assuming a linear elastic response of its constitutive material). Some precautions should be taken in order to make consistent comparisons between numerical and experimental results. Indeed, as explained previously, in experiments the contact pressure history is deduced from 

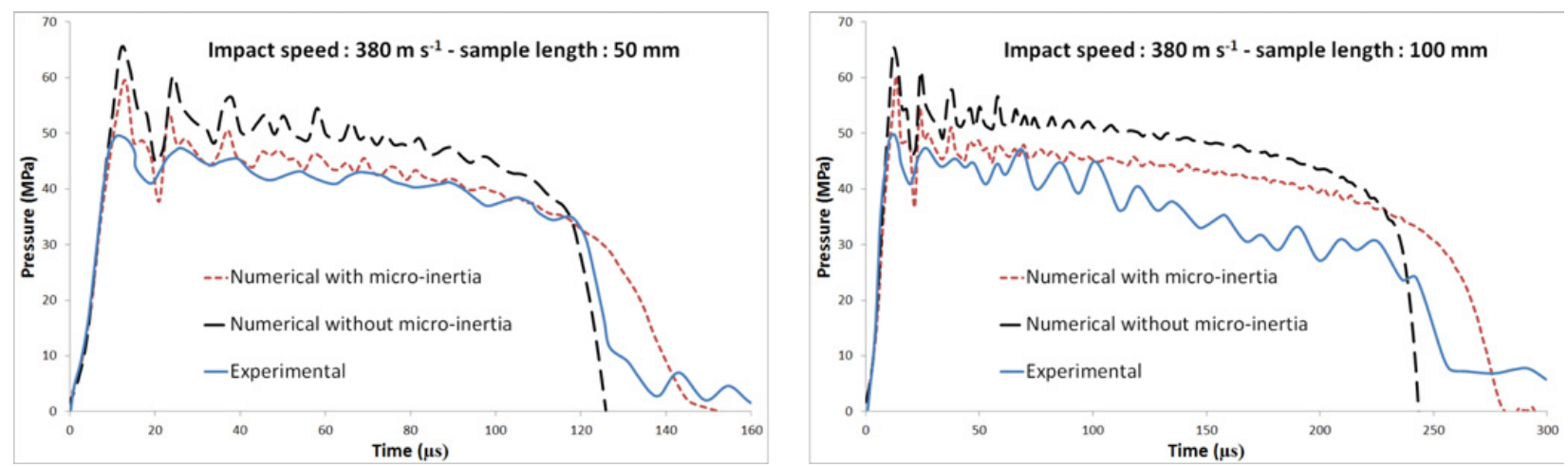

Figure 4. Influence of wave dispersion in the steel bar. This figure presents a comparison between the contact pressure history applied at the end of the bar and the stress history at $285 \mathrm{~mm}$ of this surface, as predicted by an axisymmetric Autodyn ${ }^{\circledR}$ simulation.

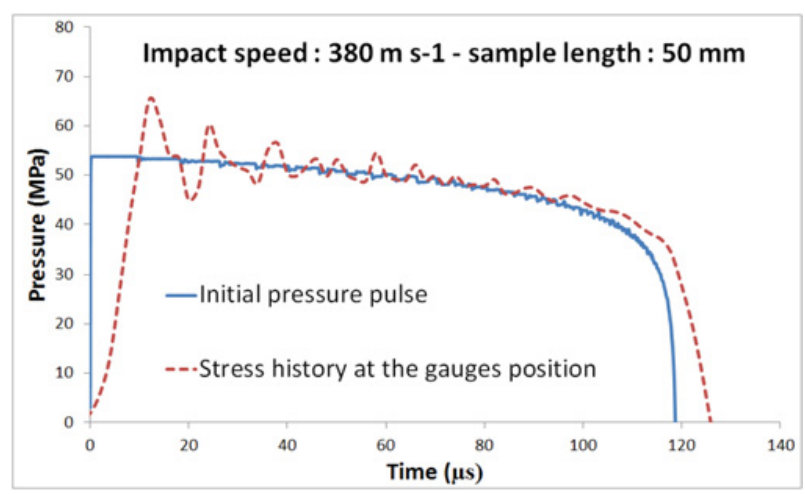

Figure 5. Impact of aluminium foam samples of lengths 50 and $100 \mathrm{~mm}$ at $380 \mathrm{~m} \mathrm{~s}^{-1}$. Contact pressure histories obtained with one-dimensional finite element computations, with and without taking micro-inertia into account, are compared to the experimental data of Radford et al. [23].

signals of strain gauges placed at about $285 \mathrm{~mm}$ of the impact surface. It is well known that the propagation of elastic waves in cylindrical bar is dispersive, meaning that some distortion of the stress pulse occurs during propagation.

In order to analyse the influence of wave dispersion in the experiments of Radford et al. [23], we have carried out axisymmetric simulations of wave propagation in the steel bar using the Autodyn ${ }^{\circledR}$ code. The contact pressure history derived from the Fortran code is applied on the end of the bar and we analyse the evolution of the stress pulse along the bar. Figure 4 shows a comparison between the pressure pulse applied on the end of the bar and the axial stress history observed at the position of the strain gauges (285 $\mathrm{mm}$ of the end). It appears that wave dispersion induces significant fluctuations of the stress. All the results presented hereafter take wave dispersion in the steel bar into account (using axisymmetric Autodyn ${ }^{\circledR}$ simulations). Let us now discuss the influence of micro-inertia. Figure 5 shows the experimental stress history and the corresponding numerical results, obtained with $\left(R_{c}=2\right.$ $\mathrm{mm})$ and without $\left(R_{c}=0\right)$ micro-inertia effects, for the impact of Alporas ${ }^{\circledR}$ foam samples of lengths 50 and $100 \mathrm{~mm}$ at $380 \mathrm{~m} \mathrm{~s}^{-1}$. Firstly, one can observe the influence

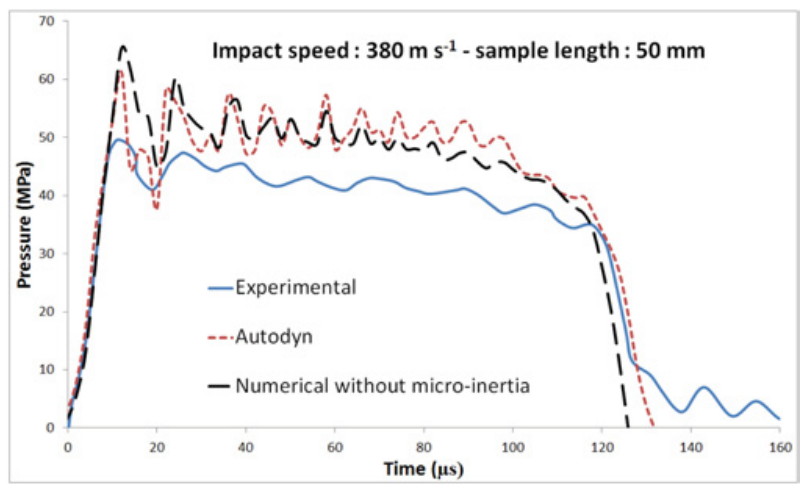

Figure 6. Comparison of experimental measurements and numerical predictions (Autodyn ${ }^{\circledR}$ and Fortran without microinertia) for the impact of an Alporas ${ }^{\circledR}$ foam sample of length $50 \mathrm{~mm}$ at a velocity of $380 \mathrm{~m} \mathrm{~s}^{-1}$.

of micro-inertia on the pressure pulse. Accounting for micro-inertia in the simulation leads to:

- a 5\% decrease in the peak and average values of the contact pressure;

- an increase of about $15 \%$ of the pressure pulse duration.

Secondly, it can be seen that numerical predictions (both with and without micro-inertia) are in a quite well agreement with experimental measurements. Nevertheless, a better correlation is achieved when micro-inertia effects are included in the modelling.

\subsection{Autodyn simulations}

For comparison purpose, we also carried some simulations of the impact tests using the dynamic code ANSYS Autodyn ${ }^{\circledR}$. In these simulations, both the foam projectile and the Kolsky bar are modelled. The foam behaviour is described by the "crushable foam" model (the version of the "crushable foam model" available in Autodyn ${ }^{\circledR}$ is the same than the one in LS-Dyna, that has been discussed by Pattofatto \& al. [15]). This model requires the axial stress-volumetric strain curve and the initial bulk and shear moduli. The Autodyn ${ }^{\circledR}$ computations do not take microscale inertia into account.

The numerical prediction obtained with Autodyn ${ }^{\circledR}$ for the impact of an Alporas ${ }^{\circledR}$ foam sample (length: 
$50 \mathrm{~mm}$ - impact velocity: $380 \mathrm{~m} \mathrm{~s}^{-1}$ ) is presented in Fig. 6, together with the experimental stress history and the result of a simulation performed with the 1D fortran code, neglecting micro-inertia. On the one hand, it appears that the Autodyn ${ }^{\circledR}$ simulation underpredicts the pressure level. On the other hand, the Autodyn ${ }^{\circledR}$ results are very close to those obtained with the 1D Fortran code. This suggests that taking micro-inertia into account in the Autodyn ${ }^{\circledR}$ simulations may improve the agreement with the experimental results.

\section{Concluding remarks}

An original model was developed to describe the response of foams under highly dynamic solicitations. Through dynamic homogenization techniques, micro-inertia effects induced by the rapid crushing of foam cells are taken into account. The macroscopic stress in the foam is the sum of a quasi-static and a dynamic (inertial) term. The dynamic stress involves only physical parameters, as it depends on the initial foam density and the mean radius of the cells of the foam microstructure.

This constitutive model was implemented using a onedimensional finite element code written in Fortran 90. Numerical simulations of the impact tests conducted by Radford \& al. [23] for aluminium closed-cell foams have been carried out. Despite some discrepancies, the results obtained with the Fortran code are in a good agreement with the experimental results when micro-inertia effects are included in the modelling.

Simulations were also conducted using ANSYS AUTODYN ${ }^{\circledR}$. The foam is modelled by using the "crushable foam" model. Micro-inertia effects are not taken into account in these simulations (it might be possible to implement the inertia the proposed modelling in Autodyn ${ }^{\circledR}$ with the use of user-subroutines). The Autodyn ${ }^{\circledR}$ results are close to those obtained by the Fortran code when no micro-inertia is included.

Future works will include the implementation of micro-inertia effects into ANSYS AUTODYN ${ }^{\circledR}$. To check and extend the validity range of the model presented in this paper, impact experiments will be conducted at velocities ranging from 500 to $2000 \mathrm{~m} \mathrm{~s}^{-1}$. Results of these tests will be the scope of another paper.

The work reported was supported by the "Association Nationale de la Recherche et de la Technologie" (ANRT) through grant CIFRE no 0843/2013.

\section{References}

[1] L. Gibson, M. Ashby, Cellular solids: Structure and properties - second edition (Cambridge University Press, 2005)
[2] M. Ashby, A. Evans, N. Fleck, L. Gibson, J. Hutchinson, H. Wadley, Metal Foams : A Design Guide (Butterworth-Heinemann, 2000)

[3] E. Andrews, W. Sanders, L. Gibson, Materials Science and Engineering A 270, 113 (1999)

[4] T. Nieh, K. Higashi, J. Wadsworth, Materials Science and Engineering A 283, 105 (2000)

[5] D. Ruan, G. Lu, F. Chen, E. Siores, Composite structures 57, 331 (2002)

[6] J. Blazy, Ph.D. thesis, École Nationale supérieure des Mines de Paris (2003)

[7] L. Gong, S. Kyriakides, International Journal of Solids and Structures 42, 1381 (2005)

[8] L. Gong, S. Kyriakides, W. Jang, International Journal of Solids and Structures 42, 1355 (2005)

[9] Z. Zou, S. Reid, P. Tan, S. Li, J. Harrigan, International Journal of Impact Engineering 36, 165 (2009)

[10] K. Dannemann, J. Lankford, Materials Science and Engineering A 293, 157 (2000)

[11] T. Mukai, T. Miyoshi, S. Nakano, H. Somekawa, K. Higashi, Scripta Materialia 54 (2006)

[12] F. Yi, Z. Zhu, F. Zu, S. Hu, P. Yi, Materials Characterization 47, 417 (2001)

[13] P. Tan, S. Reid, J. Harrigan, International Journal of Solids and Structures 49, 2744 (2012)

[14] I. Elnasri, S. Pattofatto, H. Zhao, H. Tsitsiris, F. Hild, Y. Girard, Journal of the Mechanics and Physics of Solids 55, 2652 (2007)

[15] S. Pattofatto, I. Elnasri, H. Zhao, H. Tsitsiris, F. Hild, Y. Girard, Journal of the Mechanics and Physics of Solids 55, 2672 (2007)

[16] H. Zhao, I. Elnasri, S. Abdennadher, International Journal of Mechanical Sciences 47, 757 (2005)

[17] P.A. Romero, S.F. Zheng, A.M.C. no, Journal of the Mechanics and Physics of Solids 56, 1916 (2008)

[18] A. Molinari, S. Mercier, Journal of the Mechanics and Physics of solids 49, 1497 (2001)

[19] C. Sartori, S. Mercier, N. Jacques, A. Molinari, Mechanics of Materials 80, 324 (2015)

[20] S. Reid, C. Peng, International Journal of Impact Engineering 19, 531 (1997)

[21] J. Harrigan, S. Reid, A.S. Yaghoubi, International Journal of Impact Engineering 37, 918 (2010)

[22] J. Simo, T. Hugues, Computational Inelasticity (Springer, 1998)

[23] D. Radford, V. Deshpande, N. Fleck, International Journal of Impact Engineering 31, 1152 (2005)

[24] V. Deshpande, N. Fleck, Journal of the Mechanics and Physics of Solids 48, 1253 (2000) 